\title{
La méthode chimique pour la mesure du débit des cours d'eau*
}

\section{Chemical method for the gauging of streams}

\author{
PAR HENRY DUMAS
}

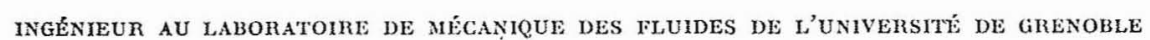

(SUITE E'T FIN)

Techniques opératoires. - Prévaration des solutions. - Techniques d'injection. - Technique des prélènements, influence de la nature des eaux. - Transporl du matériel. - But des jaugeages et mélhodes. - Résultats expérimentaux. - Conclusion.
Field practice.-Preparation of solutions.-Injection technique and devices.-Sampling technique and devices; special precantions for turbid water.-Transport of material.-Various gauging objectives and methods.-Experimental results.-Conclusion.

\section{N T R O D U C T I O N}

Dans les deux premiers chapitres de cette étude on a défini les principes de la méthode chimique et les conditions déterminant le choix du sel le plus convenable à son application, ainsi que les principes généraux d'analyse.

Le présent chapitre est consacré à la mise en œuvre de la méthode sur le terrain, à la description du matériel utilisé et à l'examen de résultats de jaugeages.

L'emploi du bichromate de soude a été seul envisagé ici.

L'expérience nouvelle de ce produit a été acquise en effet au cours des cinq dernières années et les avantages procurés par ce sel ont conduit les expérimentateurs à l'utiliser de préférence aux autres et à créer un matériel de mesure

$\left(^{*}\right)$ Cf. la Hauille Blanche, $n^{\circ} 5,1952$, p. $590 ; n^{\circ} 1$, 1953 , p. 51. bien adapté à son emploi. C'est grâce à l'appui de la Région d'Equipement Hydraulique Alpes II d'Electricité de France à Chambéry et au large crédit qu'elle fit à la méthode dès le début des recherches du Laboratoire, grâce encore au concours de son personnel et de ses moyens matériels que les techniques opératoires ont pu être amenées progressivement à leur point d'achèvement actuel. C'est ainsi que sur le vaste champ d'opérations à l'échelle industrielle offert au Laboratoire par la Région d'Equipement Hydraulique Alpes II, des jaugeages ont été exécutés couvrant une gamme de débits compris entre quelques dizaines de litres par seconde jusqu'à près de $100 \mathrm{~m}^{3} / \mathrm{s}$ et cela jusqu'à des altitudes de 2.000 mètres, dans des conditions topographiques et météorologiques très variées, éminemment propices à procurer les incidents techniques les plus divers. 


\section{A. - TECHNIQUES OPERATOIRES}

\section{I. - Préparation de la solution mère}

Tous les anciens praticiens de la méthode chimique de la période du chlorure de sodium ont connu les difficultés que présentait la dissolution de ce sel. Sa solubilité relativement faible laissait courir le risque de voir du sel non dissous rester dans les réservoirs, d'où une homogénéité imparfaite de la solution mère. Celle-ci devait donc être préparée dans des réservoirs indẻpendants de ceux qui étaient affectés à l'injection proprement dite $\left(^{*}\right)$, d'où la nécessité de pompes de transvasement. Tout ce lourd appareillage se trouvait compliqué encore par temps froid, car il devenait alors indispensable de chauffer l'eau de dissolution à l'aide de braseros. Ces inconvénients con̂teux, qui entrent pour une large part dans la désaffection qui entoura généralement la méthode chimique, résultaient principalement $d u$ fait que, pour limiter la capacité des récipients utilisés, la solution mère devait être réalisée au titre de $200 \mathrm{~g}$ et plus par litre, c'est-à-dire non loin de la saturation.

L'adoption d'un sel très soluble et dosable à un très faible titre devait donc permettre de réduire très sensiblement le volume des récipients et de préparer la solution mère dans le réservoir même destiné à l'injection, tout en écartant le risque d'une dissolution incomplète. Le bichromate de soude répond à ces conditions. Avec ce sel il est rare d'avoir à réaliser la solution mère à un titre supérieur à $200 \mathrm{~g} / \mathrm{l}$, soit trois fois moins que la saturation à la température ordinaire. En fait, la solubilité du bichromate est telle, qu'en quelques minutes on est assuré d'avoir dissout la totalité du sel. Une agitation supplémentaire réalise l'homogénéité de la solution.

Au début de l'emploi du bichromate, la première idée qui s'imposa aux praticiens, en raison mème des faibles quantités de solution utilisées, fut de conserver l'usage du vase de Mariotte, bien connu d'ailleurs dans l'histoire de la méthode chimique, qui présentait l'avantage de réunir en un seul appareil le réservoir de solution et le dispositif d'injection à débit constant.

Cependant, à cet appareil devait ètre encorc adjoint un récipient de capacité égale pour la préparation en une seule fois de la solution et une pompe de transvasement. De plus, ce récipient hermétiquement fermé ne permettait pas une surveillance constante de la solution, d'où

(*) Ces réservoirs pouvaient atteindre souvent une capacité de plusieurs mètres cubes. le risque d'enfermer dans le vase, malgré les précautions prises (crépines, filtres), des débris végétaux ou autres, risquant d'obstruer plus ou moins le diaphragme réglant l'injection.

Par ailleurs, le poids d'un vase de Mariotte de 80-100 ou $200 \mathrm{l}$ avec ses pieds supports compliquait le transport en terrain accidenté. Enfin, le calage en position verticale d'un vase qui une fois plein dépassait $100 \mathrm{~kg}$ ou $200 \mathrm{~kg}$ offrait de sérieuses difficultés sur les rives cahotiques des torrents ou en terrain meuble.

C'est pour ces raisons que l'on revint à l'ancienne technique consistant à alimenter à l'aide d'un réservoir un appareil indépendant d'injection à débit constant.

Dans ces conditions, le réservoir de solution peut être réalisé avec (par exemple) un fût métallique du commerce de prix modique et de poids réduit.

Le fût métallique à essence ou à huile de $200 \mathrm{l}$ (poids $22 \mathrm{~kg}$ ) permet de couvrir une gamme de débits très étendue en rivière de turbulence favorable. Si la gamme de débit à couvrir dans une région déterminée est relativement restreinte (jusqu'à $10 \mathrm{~m}^{3} / \mathrm{s}$ maximum par exemple) le même fût coupé en deux suffit dans la plupart des cas. Si, occasionnellement, les conditions locales exigeaient une injection prolongée, on utiliserait deux de ces fûts couplés en parallèle. Enfin le prix modique de ces récipients $\left(^{\star}\right)$ permet d'entreposer un réservoir dans chaque secteur d'opération ou même à chaque poste de jaugeage et de n'avoir plus à transporter que l'appareil d'injection proprement dit et ses accessoires (moins de $20 \mathrm{~kg}$ ) ret, éventuellement, une rampe d'injection $(17 \mathrm{~kg})$.

Pour la préparation de la solution on remplit le récipient avec la moitié ou les trois quarts de la quantité d'eau définitive, on y ajoute le bichromate en une ou plusieurs fois en agitant le mélange. La dissolution est très rapide. Tout le sel étant dissous, on complète le remplissage à la quantité voulue et l'on brasse le mélange de bas en haut et inversement avec une plaque carrée $(0,20 \mathrm{~m} \times 0,20 \mathrm{~m})$ munie d'un manche. Ce mode de brassage mélange les couches inférieures et supérieures de liquide. Au cours du jaugeage, des échantillons de solution sont pris au début, au milieu et à la fin de l'injection, ce qui permet un contrôle de l'homogénéité de la solution.

A titre d'exemple, le jaugeage d'un débit de $10 \mathrm{~m}^{3} / \mathrm{s}$ dans de bonnes conditions de turbuIence avec une injection de $20 \mathrm{mn}=1200 \mathrm{~s}$, et un débit $q=100 \mathrm{~cm}^{3} / \mathrm{s}$ et pour un titre final

(*) D'occasion : 1.000 à $2.000 \mathrm{fr}$. 
$\mathrm{N}_{2}=0,5 \mathrm{mg} / 1$ demande un poids de sel total égal à :

$\mathrm{P} g=10.000 \times 0,0005 \times 1.200=6.000 \mathrm{~g}=6 \mathrm{~kg}$ et un volume de solution de 120 litres.

\section{II. - Dispositifs d'injection à débit constant}

'Tous les dispositifs d'injection à débit constant se ramènent au principe général de l'alimentation d'un orifice sous charge constante. Dans ce domaine deux procédés $\left(^{*}\right)$ sont à re-

$\left(^{*}\right.$ Exceptionnellement et dans le cas d'un jaugeage en conduite forcée on pourrait être conduit à injecter sous pression à l'aide d'une pompe centrifuge à vitesse constante. tenir dans l'application de la méthode chimique :

a) VASE DE MARIOTte.

Le principe du vase de Mariotte est bien connu et nous n'y insisterons que pour en distinguer les avantages et les inconvénients.

$1^{\circ}$ Avantages :

- Appareil unique réunissant le réservoir de solution et le dispositif à débit constant.

_- Possibilité de réaliser l'ensemble sous un faible volume (capacité 20 à $30 \mathrm{l}$ ) dans le cas des jaugeages de petits débits (**) dans les ruisseaux turbulents.

(**) Cf. La Houille Blanche, ${ }^{\circ}$ 4, 1950, Bouvard, R.E.H., Alpes II, Chambéry.

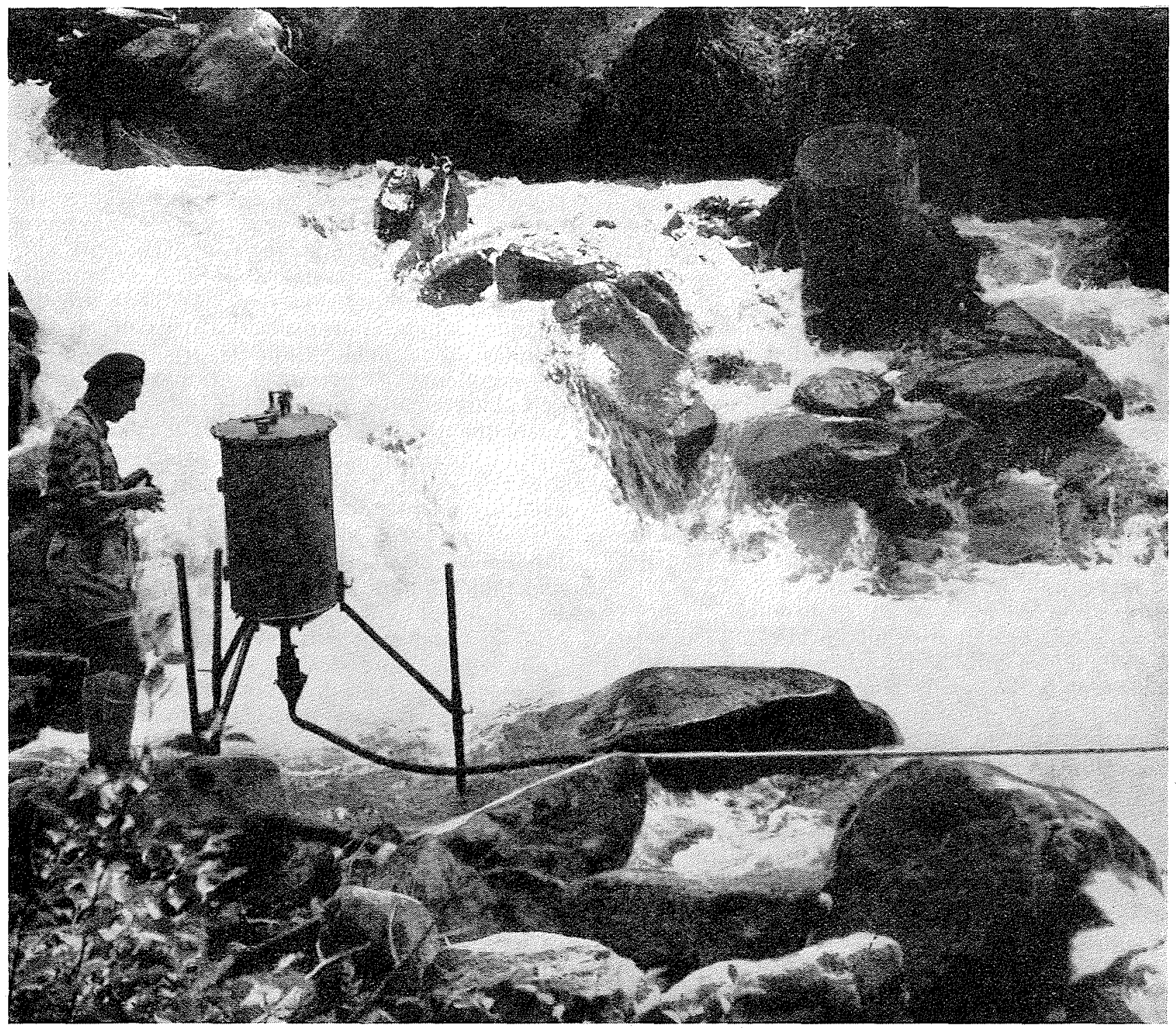

FIG. 1 


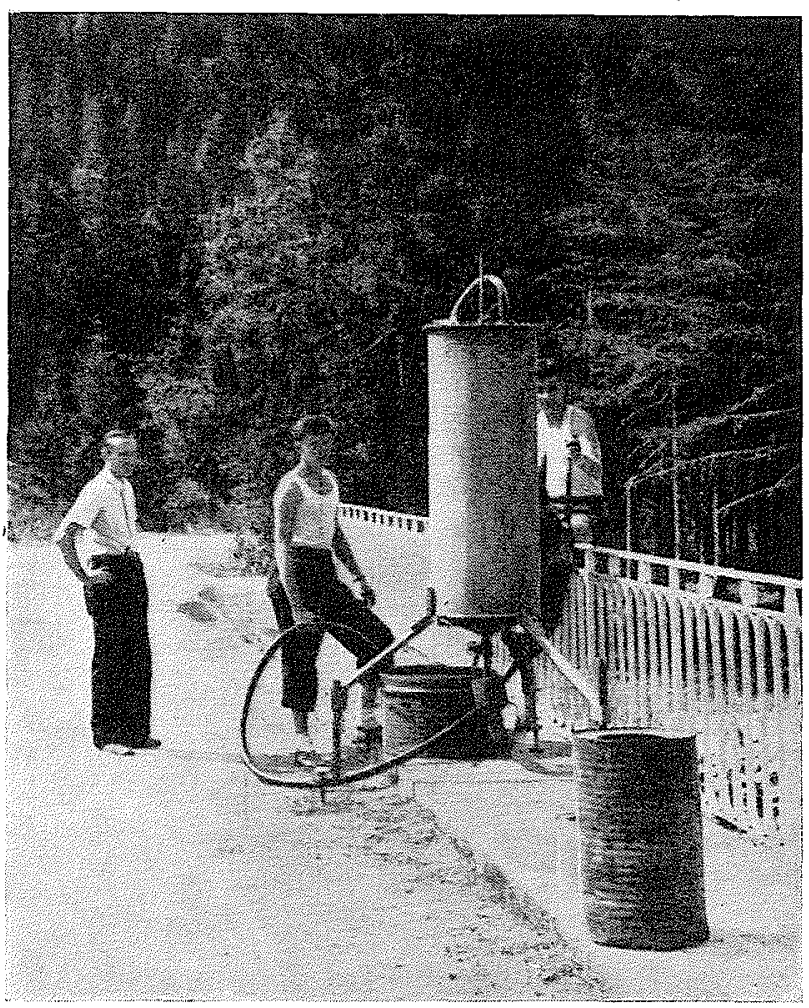

Fig. 2

\section{$2^{\circ}$ Inconvénients :}

- Poids et encombrement importants lorsque le vase de Mariotte dépasse une capacité de 100 litres.

- Installation difficile en terrain varié.

- Nécessité d'un récipient d'égale capacité pour la préparation de la solution.

- Nécessité de transvaser la solution mère dans le vase de Mariotte, d'où l'emploi de pompes.

- Dans l'opération du transvasement, risques de débordements et de pollution du cours d'eau avant le jaugeage.

- Nécessité d'obtenir une bonne étanchéité du vase, d'où présence de joints dont le serrage imparfait risque de produire des entrées d'air suffisantes pour troubler la constance du débit.

- Impossibilité de contrôler la pureté de la solution dans le vase.

- Fig. 1 : vase de Mariotte de $80 \mathrm{l}$ (Laboratoire) *.

-- Fig. 2 : vase de Mariotte de 200 l. (E.D.F. R.E.H. Alpes II) ${ }^{* \star}$.

(*) Jaugeage du Versoyen (Savoie), R.E.H. Alpes II.

${ }^{*}$ *) Jaugeage de l'Arveyron à Chamonix, R.E.H. AIpes II. b) VAse a niveau constant.

Le dispositif se compose (fig. 3 ret 4) d'un diaphragme interchangeable situé à la base d'un tube large $(80 \mathrm{~mm})$ surmonté d'une capacité comportant un déversoir. Le vase est alimenté par un réservoir supérieur par l'intermédiaire d'un robinet de réglage très progressif fonctionnant par écrasement du tuyau de caoutchouc d'adduction. Au fur et à mesure de la vidange du réservoir, le robinet est ouvert peu à peu, de manière à maintenir une très faible surverse sur le déversoir. Celle-ci est récupérée dans un seau et reversée dans le réservoir supérieur.

Un clapet permet de fermer le diaphragme et de l'ouvrir au moment voulu.

Un entonnoir inférieur orientable conduit la solution dans les tuyaux d'injection.

Un filtre placé dans la capacité supérieure arrête les corps étrangers qui pourraient obstruer le diaphragme. Enfin, un système de pieds réglables permet de placer le vase en position verticale quelle que soit la configuration du terrain.

Poids du vase et de ses accessoires: $15 \mathrm{~kg}$.

Fig. 5 : vase à niveau constant et son réservoir supérieur ** ${ }^{*}$

$\left({ }^{* * *}\right)$ Jaugeage du Cormet d'Arèches (Savoie). E.D.F., R.E.H. Alpes II,

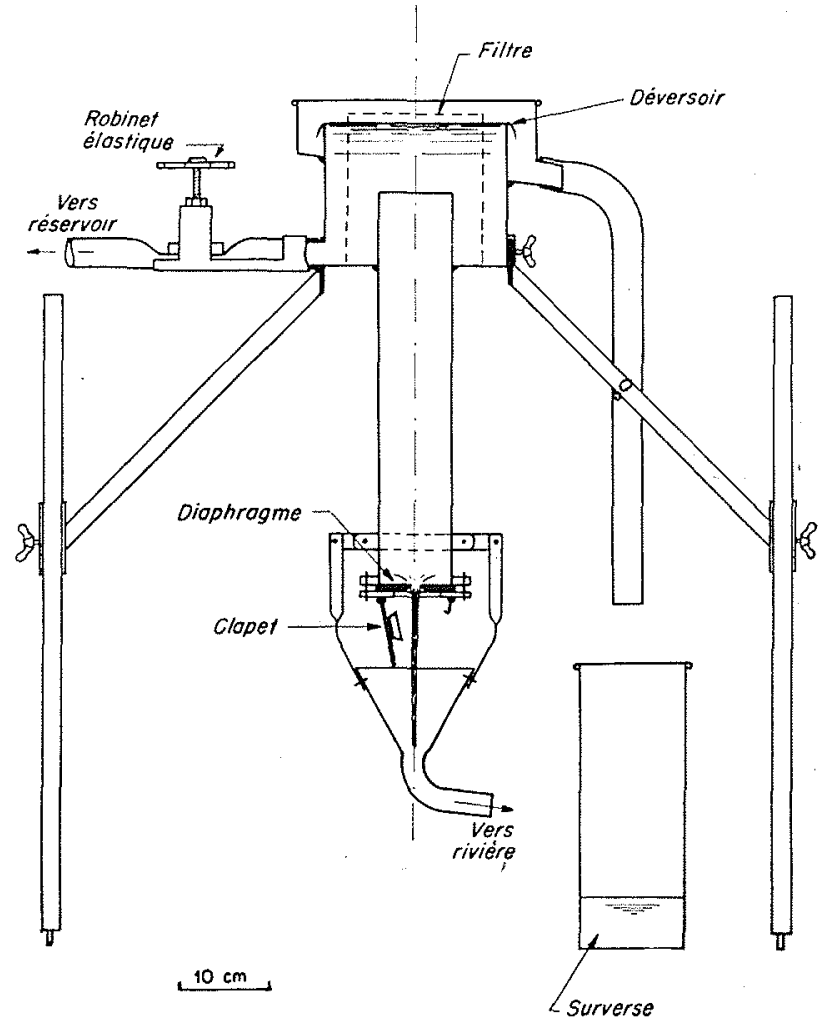

FiG. 3 
Les cinq diaphragmes utilisés couvrent une gamme de débiț d'injection allant de $25 \mathrm{~cm}^{3} / \mathrm{s}$ à $225 \mathrm{~cm}^{3} / \mathrm{s}$ environ.

Utilisé avec un réservoir de 200 litres, l'appareil permet de réaliser des temps d'injection compris entre 5 minutes et 2 heures environ.

\section{A vantages}

- Poids négligeable et encombrement réduit.

- Contròle permanent de la pureté de la solution.

- Absence de transvasement de solution d'où suppression des pompes.

- Possibilité d'entreposer sur les lieux d'opération le ou les réservoirs principaux et de réduire le transport à celui de l'appareil d'injection.

- Contrôle permanent de la constance du débit d'injection.

Dans un cas comme dans l'autre les divers diaphragmes utilisés sont étalonnés à l'avance de préférence par pesée. Sur place un contrôle plus grossier du débit, destiné uniquement à vérifier que le diaphragme convenable a bien été employé, se fait en mesurant le temps glo-

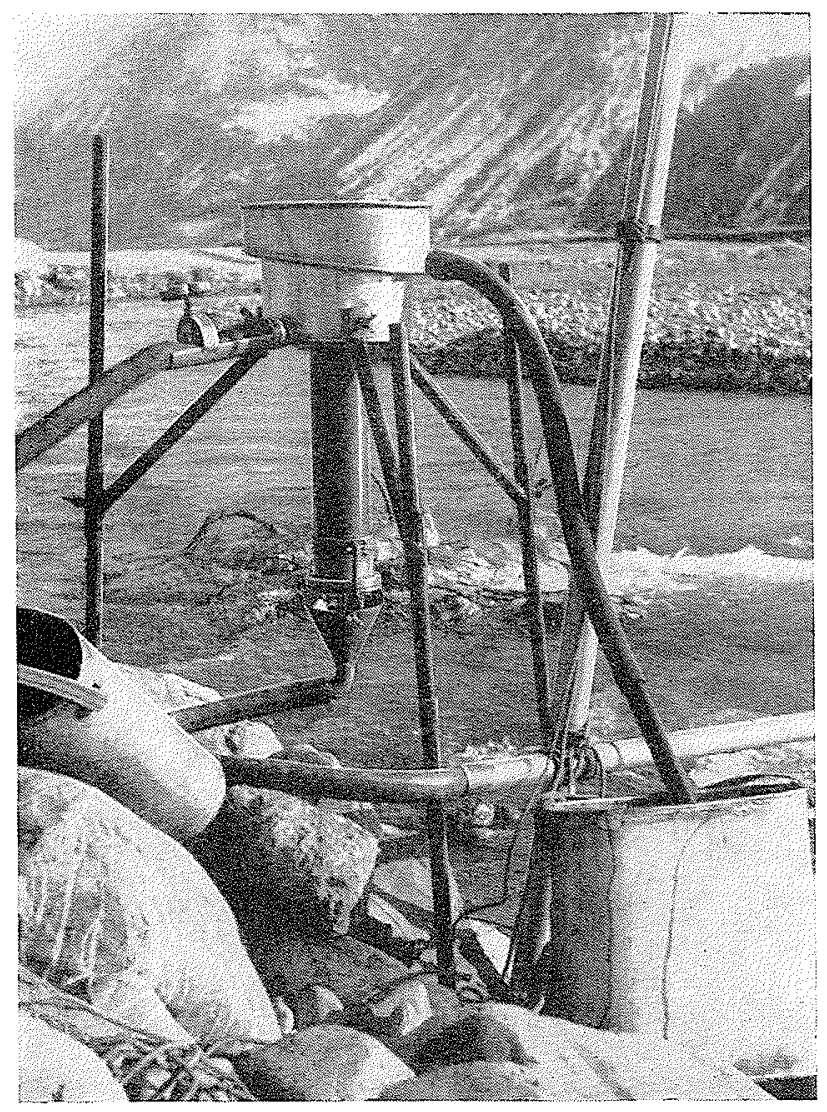

FIG. 4 bal de vidange de la quantité totale de solution utilisée.

Le procédé du vase à niveau constant s'impose pour les séries de jaugeages demandant une grande mobilité de manœuvre et pour les régions accidentées. La grande rusticité de cet appareil, son déréglage pratiquement impossible le rendent particulièrement adapté aux transports quelque peu violents. Dans certains cas où des installations à poste fixe seraient prévues, d'autres techniques d'injection peuvent être envisagées.

c) Appareils divers.

Au nombre de ceux-ci on peut noter :

- Le siphon rigidement lié à un flotteur qui permet d'injecter à partir du réservoir de solution sans l'intermédiaire d'un vase à niveau constant. Cet appareil nécessite un calage bien vertical et un guidage précis du flotteur. L'amorçage du siphon est une difficulté.

- Le réservoir à niveau constant en moyenne alimenté depuis la réserve de solution par l'intermédiaire d'un robinet commandé par un flotteur. Ce dispositif particulier aux postes fixes permet à un seul opérateur de déclencher l'injection qui se poursuit seule pendant qu'il va recueillir les échantillons dans la section de prélèvement. Le débit moyen d'injection est alors indiqué par un limnigraphe qui enregistre en fonction du temps l'abaissement de la solution mère dans la réserve $\left(^{\star}\right)$.

\section{III. - Dispositifs d'adduction de la solution au cours d'eau}

La solution qui s'écoule par le diaphragme de mesure est conduite à la rivière par des tuyaux d'adduction.

Deux méthodes sont applicables suivant les conditions locales :

a) Injection ponctublate (fig. 1-5).

Dans les torrents de montagne très turbulents la répartition latérale de la solution est complète et s'accomplit rapidement sur une faible longueur de parcours. Dans ces conditions, on peut déverser la solution dans la rivière à l'aide d'un tuyau dont l'extrémité est placée à l'aplomb d'une zone particulièrement turbulente de l'écoulement. Il est généralement préférable de laisser la solution s'écouler à l'air

(*) Installation du Service d'Etudes et Recherches E.D.F. sur la Souloise, la Sévelnisse, le Drac (Isère). 


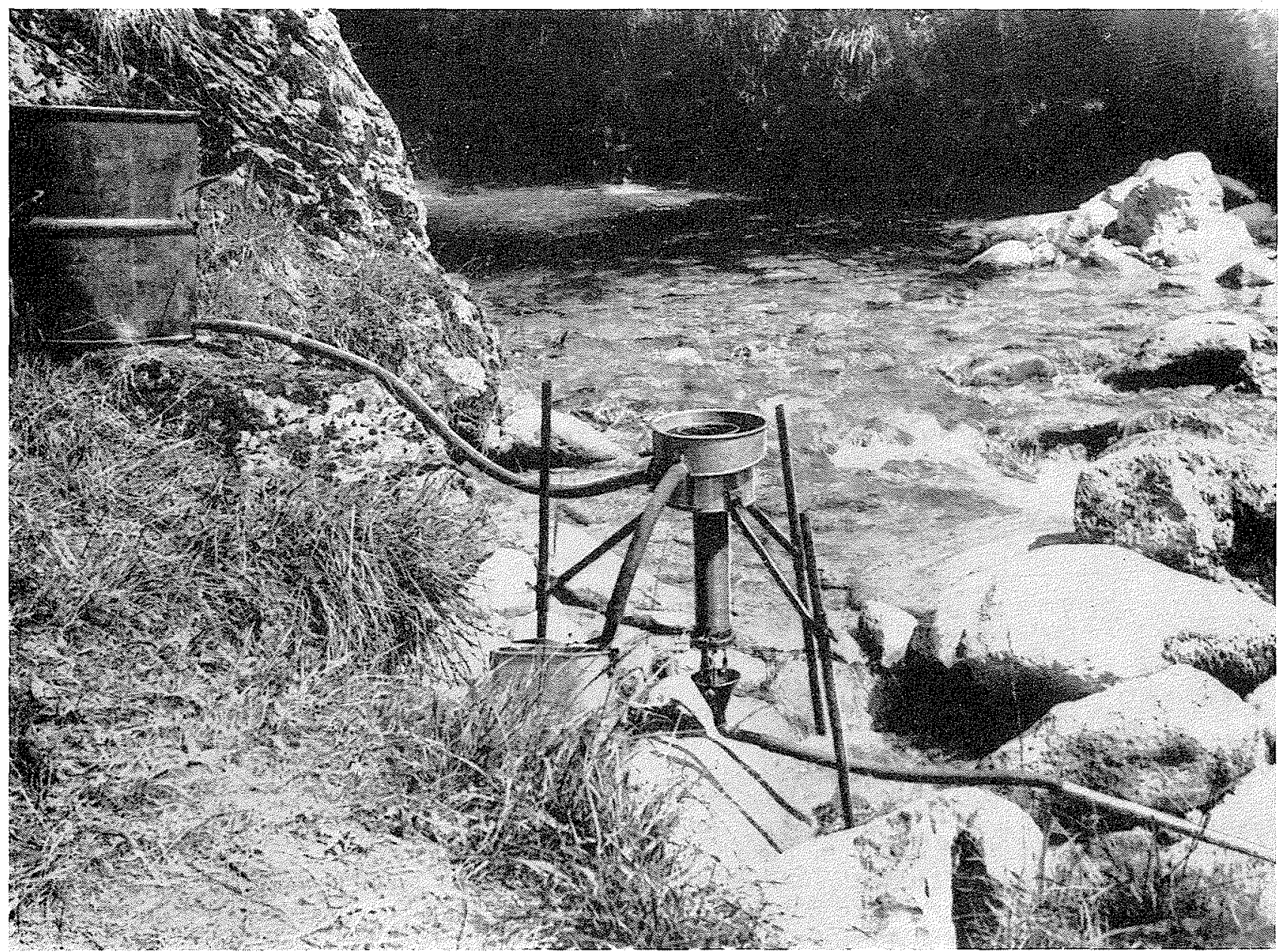

FIG, 5

libre au-dessus de l'écoulement. L'immersion de l'extrémité du tuyau dans l'eau de la rivière risque, en effet, de troubler la constance du débit de la solution mère du fait des refoulements qui pourraient se produire dans le tuyau par suite de la turbulence.

Dans des cours d'eau plus étalés où la turbulence est souvent moins active en apparence, l'injection ponctuelle peut encore se pratiquer si l'essai colorimétrique préalable a montré qu'à partir d'une certaine distance du point d'injection la répartition latérale s'établit convenablement dans toute la section de la rivière.

\section{b) INJECTION RÉpARTIE.}

Dans le cas de rivières à turbulence relativement fine, on a souvent intérêt à répartir la solution mère en travers de l'écoulement, de manière à provoquer dès le départ la formation d'un nuage intéressant toutes les masses d'eau de la section transversale. L'expérience montre que la longueur du secteur nécessaire pour obtenir un bon mélange peut être moins grande qu'avec l'injection ponctuelle. La solution est déversée dans l'écoulement à l'aide d'une rampe de tuyaux perforés qu'il est commode de lancer en travers de la rivière à l'aide d'un mât et de cordages de suspension. Le dispositif (fig. 6) permet de régler la rectitude et la pente de la rampe. Avec un mât télescopique de 5 mitres, on peut lancer avec facilité une rampe de 14 mètres de longueur. Ce système présente l'avantage de permettre les jaugeages à partir d'une seule des rives du cours d'eau (fig. 7) (*).

Poids de la rampe de $14 \mathrm{~m}$ avec son mât de lancement, cordages et accessoires : $15 \mathrm{~kg}$.

Nous rappelons ici que le mode d'injection qui est lié à la nature de l'écoulement localisé au point d'injection l'est aussi à l'aspect du secteur de mesure considéré dans son ensemble. Il serait inadéquat, en effet, de faire une

(*) Jaugeage du torrent des glaciers, R.E.H., Alpes II. 
injection distribuée uniformément dans la section transversale si le débit était réparti à $90 \%$ sur un tiers de la largeur et à $10 \%$ sur les deux tiers restants (rivières présentant des coudes et un profil en travers triangulaire). De telles conditions imposent d'ailleurs le choix d'un autre secteur de mesure. On aura toujours présentes à l'esprit les conditions de choix de ce secteur :

$1^{\circ}$ Secteur aussi rectiligne que possible.

$2^{\circ}$ Lit prismatique et profil en travers à peu près symétrique.

3: Transit de l'eau et turbulence aussi intenses que possible en tous points de la section transversale.

$4^{\circ}$ Absence de zones d'eau morte (bassins latéraux ou mouilles profondes).

\section{IV. - Technique des prélèvements}

\section{a) MatÉriel.}

Les échantillons recueillis dans la rivière dans la section de prélèvement sont de faible volume $\left(90 \mathrm{~cm}^{3}\right)$. L'usage a démontré la commodité d'emploi des flacons à large goulot dits "poudriers» pourvus d'un bouchon fileté métallique ou en matière plastique. $\mathrm{Vu}$ le faible poids de ces flacons, il est facile de les fixer à l'extrémité d'une perche à rallonge en dura- lumin dans un capot muni d'un ressort d'arrêt (fig. 8). La perche de prélèvement et sa rallonge permettent de recueillir l'eau à $5 \mathrm{~m}$ de distance (fig. 9). Les flacons sont transportés dans une caissette de 16 flacons dont 2 sont réservés aux prélèvements de solution mère. (Poids d'une caissette pleine : $5 \mathrm{~kg}$ ).

Les bouchons à vis ne doivent pas comporter de rondelle d'étanchéité en liège à cause de la coloration que pourrait apporter le tanin et du risque de voir des traces de bichromate retenues dans le liège après emploi. Ces rondelles sont remplacées par des rondelles de papier paraffiné changées chaque fois.

Enfin, les flacons de solution mère sont nettement différenciés des flacons de prélèvement, les bouchons et le corps des flacons étant marqués de façon très apparente. Ainsi on évitera de prendre pour les prises d'échantillons dilués des flacons qui pourraient conserver des traces de solution concentrée.

Le matériel qui vient d'être décrit permet de faire des prélèvements instantanés en différents points de la surface de l'écoulement. Dans certains cas on peut être amené à contrôler l'homogénéité du mélange final en faisant des prélèvements en profondeur. On peut aussi vouloir faire un prélèvement moyen en étalant le remplissage du flacon sur une certaine durée (30 secondes-1 minute). L'ingéniosité des opérateurs s'est ainsi exercée à créer des systèmes à clapet permettant d'ouvrir le flacon à toute profondeur voulue ou à diaphragmes calibrés ré-

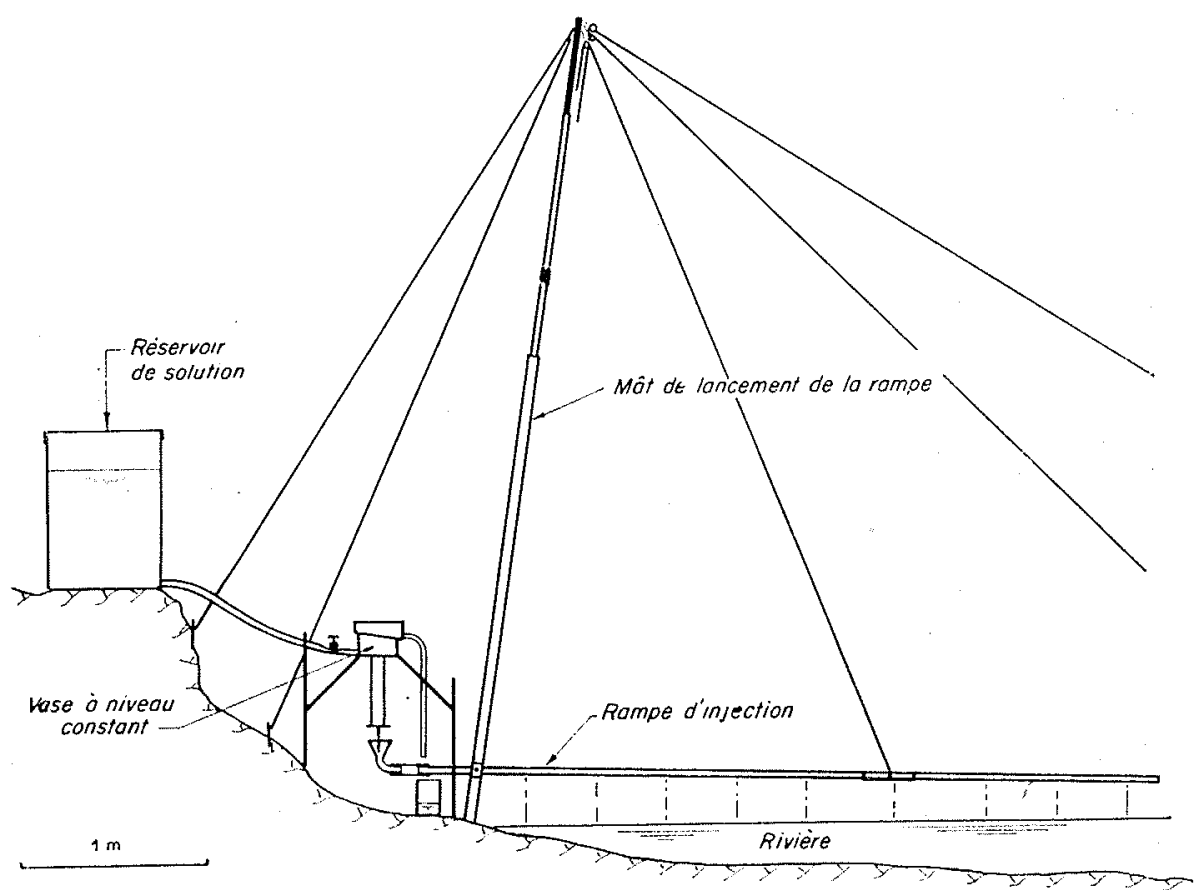

Fic. 6 


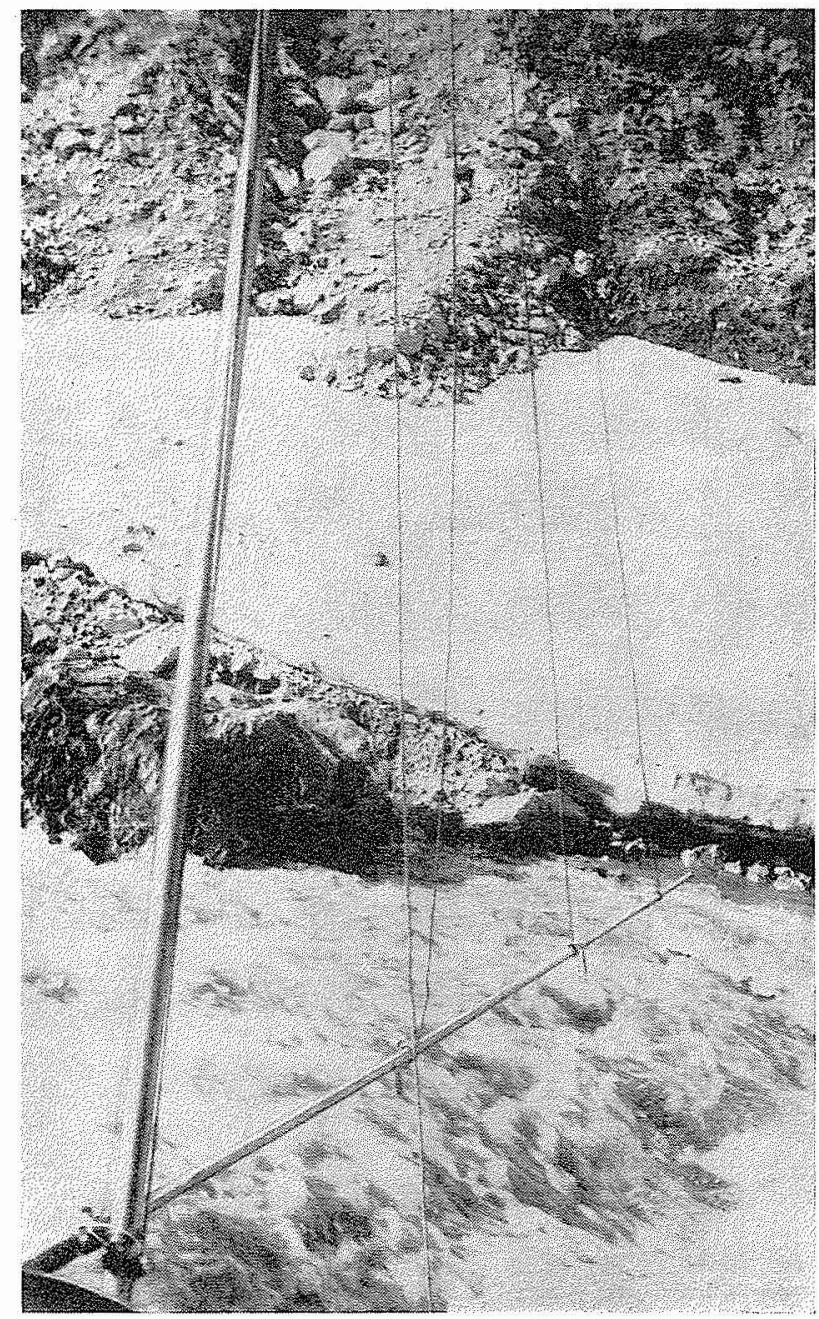

Fig. 7

glant le remplissage en un temps déterminé, l'air contenu dans le flacon étant évacué par l'intermédiaire de la perche de manœuvre.

Dans tous les cas, les prélèvements sont exécutés à intervalles régulièrement répartis dans la durée prévue du régime permanent.

Au cours du jaugeage, les flacons sont pris dans leur ordre de numérotation. L'heure et l'emplacement de la prise sont notés pour chacun d'eux.

\section{b) Nature DES EAUX.}

Les eaux des torrents peuvent être claires ou troubles. Si elles sont claires, l'analyse colorimétrique peut ètre exécutée sans risques en préparant l'échelle de dilution de la solution mère, à partir de l'eau du laboratoire. Si elles sont troubles, deux cas se présentent :

- 1" L'eau se décante rapidement (sables) et il ne reste, après une heure ou deux de décantation, aucune trace de trouble. Dans ce cas on opère comme avec les eaux claires. On peut aussi, pour gagner du temps, filtrer les échantillons sur un filtre de verre.

- $2^{\circ}$ Après décantation prolongée il reste un léger louche provenant de matières quasi colloïdales demeurant indéfiniment en suspension et passant à travers les filtres ordinaires. Il devient alors nécessaire de collecter avant le jaugeage une dizaine de litres de l'eau de la rivière qui, une fois décantée, servira à préparer l'échelle de dilution au laboratoire. Les échantillons et les solutions étalons seront ainsi dans le même état initial avant d'être présentés au colorimètre photo-électrique.

\section{V. - Transport du matériel}

Le matériel complet emballé pour le transport par chemin de fer ou autocar comprend (fig. 10):

La rampe d'injection, mât de manœuvre et deux perches de prélèvement, contenus dans une housse.......... $17 \mathrm{~kg}$

Le matériel d'injection contenu dans une caisse ................. $38 \mathrm{~kg}$

1 caisse de prélèvements (pleine).... $5 \mathrm{~kg}$

Total ............. $\overline{60 \mathrm{~kg}}$

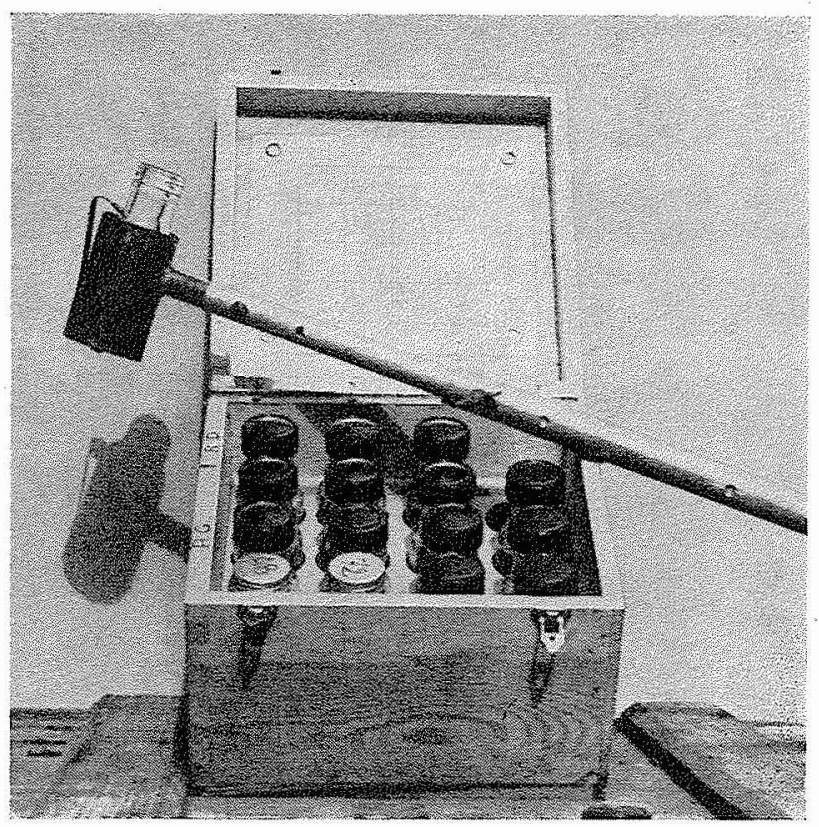

FIG. 8 
Le bichromate de soude nécessaire est en général livré en fût, de 70 à $80 \mathrm{~kg}$. On l'entrepose au centre des opérations où il est fractionné. Il $y$ a donc lieu d'ajouter aux poids ci-dessus le poids du bichromate utilisé.

Le matériel peut être transporté en vrac dans une voiture de chantier (Jeep) ou de tourisme. Dans ce cas, la suppression de la caisse de transport du vase à niveau constant fait gagner $15 \mathrm{~kg}$. Pour l'acheminement à dos d'homme, le matériel peut se diviser en parts facilement transportables.

Comme nous l'avons dit plus haut, le réservoir de solution (fût métallique) est entreposé à l'avance à proximité des stations de jaugeages.

Le transport du bichromate se fait pratiquement à l'aide de seaux tronconiques (seaux à confitures ou à miel) sans rebords, qui ont

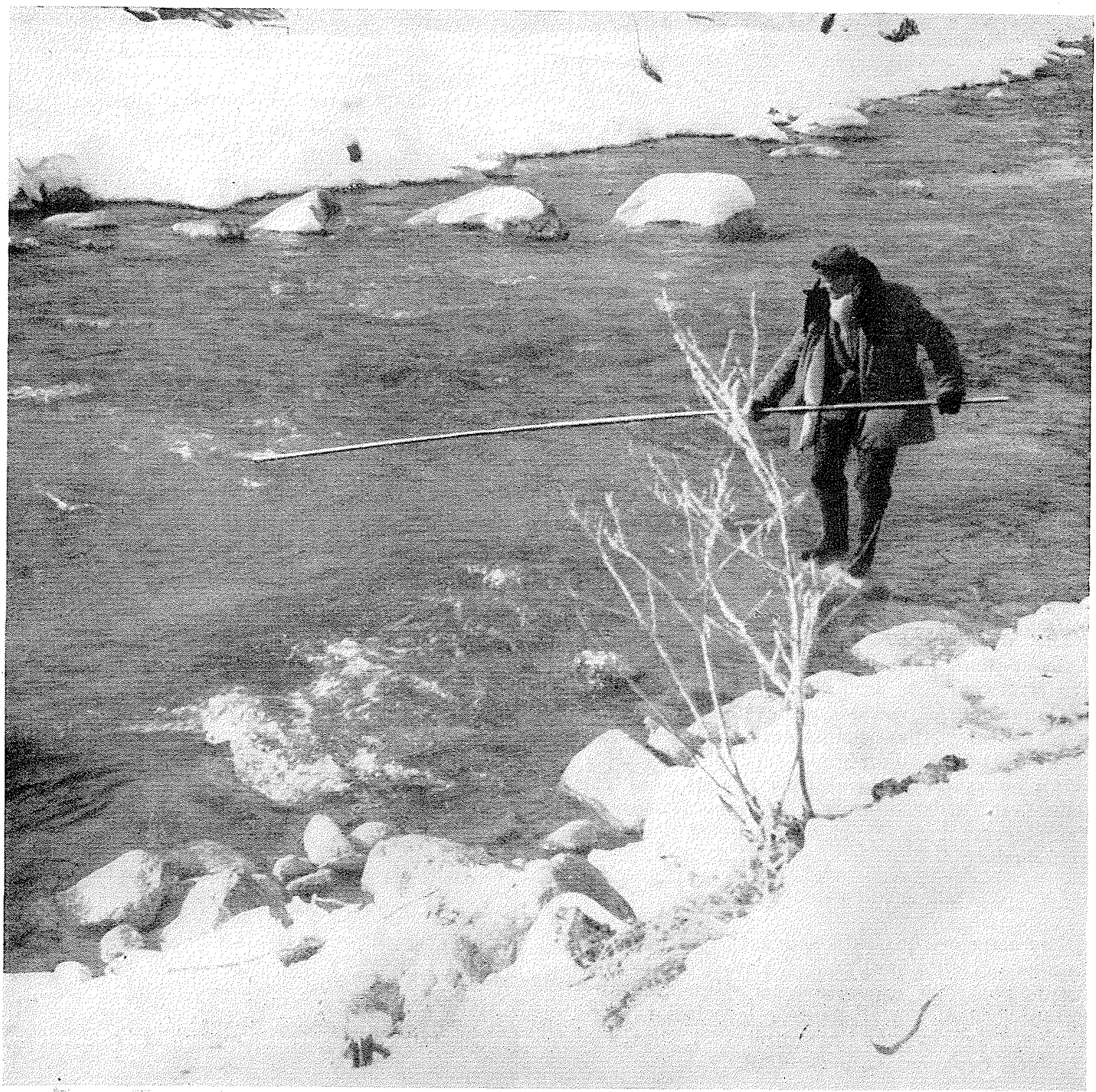

Fig. 9 


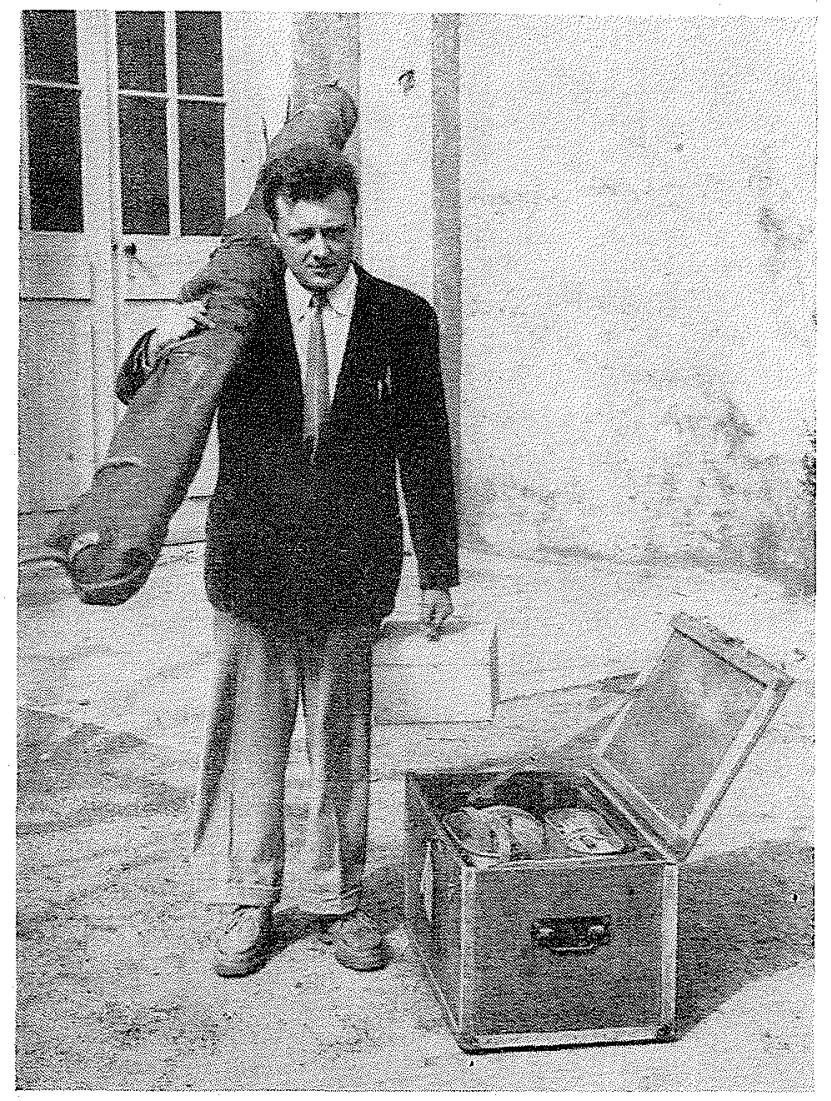

Fif. 10

l'avantage de se vider complètement. Il est commode de préparer à l'avance des quantités de bichromate pesées suivant l'échelonnement des boìtes de poids $(1-2-5)$, ce qui permet de réaliser tous les poids de sel nécessaires sans avoir de pesées à exécuter sur le terrain.

\section{B. - LES DIVERS TYPES DE JAUGEAGES}

L'objet des jaugeages de rivières est le plus souvent d'établir, pour une station donnée, la relation entre le débit et les hauteurs lues sur l'échelle de station ou enregistrées par un limnigraphe. Les jaugeages sont alors localisés aux abords de la station. Le secteur d'opération peut être déplacé dans le voisinage de la station suivant la nature de l'écoulement aux divers débits et c'est l'avantage de la méthode chimique de permettre un choix du lieu le plus favorable aux mesures.
La méthode du moulinet appliquée aux rivières demande au contraire le choix minutieux d'un emplacement unique pour les jaugeages et souvent telle section réputée satisfaisante pour un débit moyen ne le sera plus pour les débits plus faibles ou plus élevés.

Une autre application du jaugeage chimique, qui prend alors le nom de « jaugeage continu» est la détermination d'une différence de débit entre deux points d'une rivière $\left(^{\star}\right)$. Ces différences peuvent être dues à des résurgences d'écoulement souterrains ou aux apports naturels du bassin versant. La méthode chimique est particulièrement apte à la résolution de ce problème. Dans ce cas une injection prolongée est faite en un point de la rivière et des prélèvements sont exécutés à diverses abscisses à partir de ce point. Tout apport d'eau sur le parcours du nuage augmente la dilution. Il sera, bien entendu, nécessaire, par un essai colorimétrique préalable, de déterminer les durées de parcours du nuage, desquelles on déduira la durée du régime permanent à chaque section de prélèvement. Les durées d'injection peuvent être de plusieurs heures si le secteur de mesure atteint plusieurs liilomètres et si la vitesse moyenne est faible. La méthode ainsi appliquée évite la totalisation des erreurs qui risque de se produire si des jaugeages indépendants sont exécutés. Il est bien entendu nécessaire de choisir une période où le débit se maintient stable pendant la durée prévue pour les opérations.

On peut ainsi déterminer :

- L'origine des différences de débit (résurgences ou apports naturels du bassin versant) $\left({ }^{\star \star}\right)$.

- L'importance relative des apports d'un bassin versant donné en fonction de l'altitude et à des époques différentes de l'année $(* \star \star)$.

- Le débit de résurgences connues par un jaugeage différentiel entre deux points $\left({ }^{\star \star \star}\right)$.

(*) Cf. la Houille Blanche, $\mathrm{n}^{\circ} 3,1946$, Goguex : Jaugeage continu du Guil.

(**) Jaugeages du Verdon à Ste-Croix, R.E.H., Alpes III, Marseille. Alpes II,

Jaugeages de l'Are à Lanslebourg (Savoie), R.E.H.

$\left({ }^{* \star}\right)$ Jaugeages périodiques mensuels du torrent de Naves (Isère), R.E.H., Alpes II.

$\left({ }^{* * *}\right)$ Jaugeages du Borne (Hte-Savoie), R.E.H., Alpes II. 


\title{
C. - RÉSUltats deS JAUGEAGES
}

\author{
EXEMPLE I
}

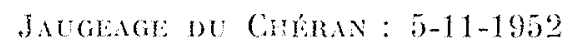

R.E.H., Alpes II

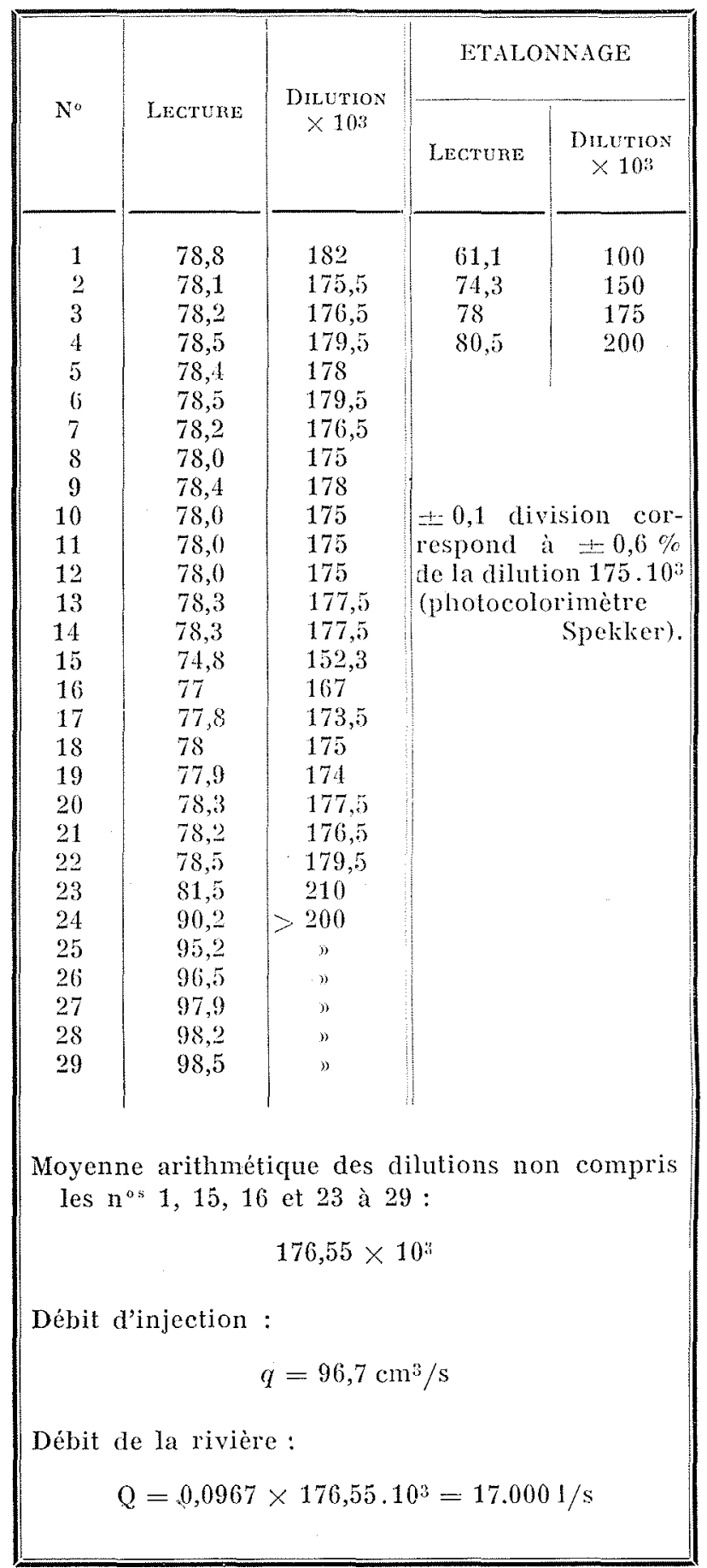

A la réception des échantillons, le laboratoire prépare l'échelle des dilutions ctalons à partir de la solution mère. Les échantillons sont alors comparés à l'échelle de dilution et le laboratoire foumit :

-.- La courbe des lectures au colorimetre en fonction des dilutions étalons.

- Les lectures au colorimètre pour chaque echantillon.

L'examen de ces résultats montre que l'on a encadré le régime permanent qui va des Nos 2 à 22 .

Le $\mathrm{N}^{\circ} 1$ (dilution $182.10^{3}$ ) est supérieur de $3 \%$ environ à la moyenne arithmétique. Les Nos 23 à 29 régulièrement croissants marquent le début de la disparition du nuage. On a négligé les Nos 15 et 16 qui sont un exemple de points erratiques que l'on peut rencontrer dans le palier de régime permanent.

Les causes de ces points erratiques sont mal connues. Ils peuvent résulter d'un nettoyage imparfait de quelques flacons de prélèvements qui conserveraient ainsi des traces de bichromate. Il est toutefois improbable, comme c'est le cas présent, que deux échantillons s'écartent consécutivement pour cette cause du groupement général des points du palier. Les écarts pourraient être recherchés dans une modification du régime d'injection. Par exemple, un réglage de l'inclinaison de la rampe d'injection au cours du jaugeage troublerait le régime permanent établi dans cette rampe et provoquerait un afflux momentané de solution concentrée dans la rivière. Dans le jaugeage pris pour exemple, le caraclère systématique et momentané des deux points erratiques permet leur suppression sans risque. Ces deux points pris en compte dans le calcul de la moyenne n'abaisseraient d'ailleurs celle-ci que de $1 \%$ environ.

On peut remarquer que la courbe d'étalonnage est établie ici dans des limites trop larges. La précision de mesure aurait été améliorée en établissant d'après les résultats ci-dessus une nouvelle courbe de dilution dilatée entre les valeurs 150 et $180 \cdot 10^{3}$.

Les écarts maxima et minima par rapport à la moyenne ressortent a :

\begin{tabular}{lrc} 
& Dicutron & Ecarts \% \\
Maximum : & $179,5 \cdot 10^{*}$ & $+1,67$ \\
Moyenne : & $176,55 \cdot 10^{3}$ & \\
Minimum : & $173,5 \cdot 10^{3}$ & $-1,73$ \\
\multicolumn{2}{c}{ Nombre de points : 20} &
\end{tabular}




\section{DILUTION ETALON}

Erreur sux la dilution... $\pm 0,5 \%$

$\because \%$

Erreur de lecture...... $\pm 0,6 \%$

Total........... $\pm 1,1 \%$

\section{Echanthluons}

Erreur de lecture :

$$
\frac{ \pm 0,6}{\sqrt{20}}=-1=0,13 \%
$$

Erreur de pointé sur la courbe :

$$
\frac{ \pm 0,2}{\sqrt{20}}= \pm 0,04 \%
$$

Total. . . . . . . . . . $\ldots 0,17 \%$

Erreur sur la moyenne........ $\pm 1,27 \%$

Erreur sur le débit d'injection.... $\neq 0,2 \%$

Erreur maximum sur le débit.... $\pm 1,5 \%$
Le jaugeage pris pour exemple montre la necessité d'encadrer le régime permanent aussi largement que possible. En effet, si les prises d'échantillons avaient été retardées par suite d'une erreur dans la détermination des temps de propagation et commencées seulement à parLir du $\mathrm{N}^{\circ} 15$ par exemple, on aurait constaté une croissance régulière des dilutions. Il aurait été logique d'en conclure que l'on se trouvait dans la période de disparition du nuage. Une extrapolation inconsidérée aurait alors situé le palier aux environs de $150.10^{3}$, soit à $15 \%$ audessous du palier réel.

EXEMPI.E II.

JaUgeage dU Reclus (Savole) : $15-5-1952$

R.E.H., Alpes II

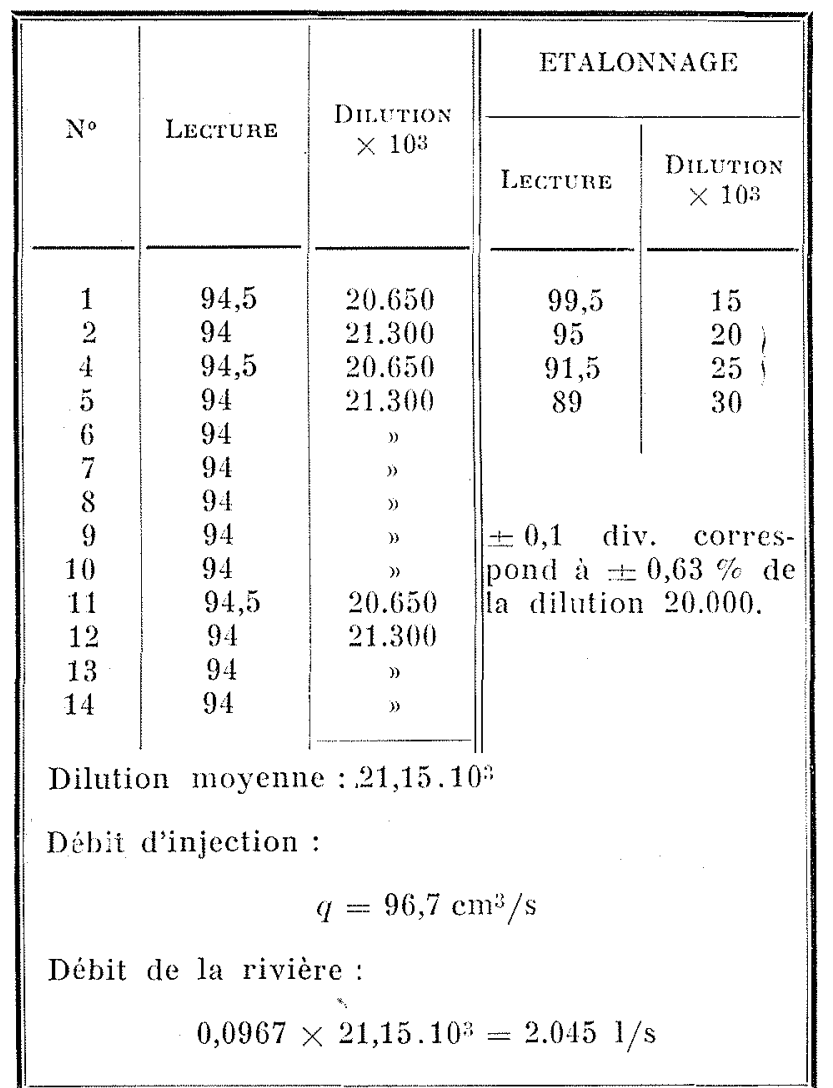

Les prélèvements ont été exécutés dans les limites du régime permanent. Trois points s'écartent de - 2,4\% de la moyenne qui ne se trouverait diminuée que de $0,7 \%$ s'il était fait abstraction de ces points.

Nombre de points: 14.

\section{Dimutions Etalons}

Erreur sur la dilution... $\pm 0,5 \%$ Erreur de lecture..... $\pm 0,63 \%$

Total ............ $\pm 1,13 \%$

\section{Echantullons}

Erreur de lecture :

$$
\frac{ \pm 0,63}{\sqrt{14}}= \pm 0,17 \%
$$

Erreur de pointé sur la courbe:

$$
\frac{ \pm 0,2}{\sqrt{14}}= \pm 0,05 \%
$$

Total ............

Erreur sur la moyenne........ $\pm 1,35 \%$

Erreur sur le débit d'injection.... $\pm 0,2$ \%

Erreur maximum sux le débit.... $\pm 1,55 \%$ 
Les exemples donnés ci-dessus caractérisent des jaugeages satisfaisants. Dans de nombreux cas le palier de régime permanent ne présente même aucune dispersion $\left(^{*}\right)$. Le premier exemple cité a été choisi comme un cas de présence de points erratiques sans incidence grave sur le calcul du débit.

Des anomalies plus sérieuses peuvent être constatées. Ce sont par exemple :

$1^{\circ}$ Variation continue du régime permanent de dilution sans que ce fait puisse être attribué à une erreur sur l'instant des prises d'échantillons (par exemple prises faites dans la période d'apparition ou de disparition du nuage).

\section{Causes}

a) Dilutions croissantes :

- Augmentation progressive du débit de la rivière.

- Non-homogénéité de la solution mère.

- Dans le cas d'emploi d'un vase de Mariotte : entrée d'air autre que l'adduction normale.

\section{b) Dilutions décroissantes:}

- Diminution progressive du débit de la rivière.

- Non-homogénéité de la solution mère.

2" Dissymétric de répartition des dilutions dans la section transversale de prise des échantillons.

\section{Causes}

a) Turbulence insuffisante de l'écoulement.

b) Courants préférentiels.

c) Présence d'une résurgence insoupçonnée sur l'une des rives du cours d'eau.

d) Insuffisance de longueur du secteur de mesure.

$3^{\circ}$ Existence de deux paliers de régime permanent.

\section{Causes}

a) Obstruction partielle à un instant donné du diaphragme réglant l'injection.

(*) Aux approximations près. b) Non-homogénéité de la solution mère, surtout dans le cas d'emploi de deux réservoirs jumelés.

Il résulte de ces faits que, dans l'ensemble, les mesures de débit des cours d'eau par la méthode de dilution demanderont en général l'exécution de quelques jaugeages préliminaires d'étude. Les visites de reconnaissance des lieux, nécessaires pour choisir en gros un secteur favorable, ne laissent pas toujours, en effet, le temps d'examiner en détail les cours d'eau par un cheminement d'exploration le long de rives souvent accidentées et de localiser à coup sûr, au premier abord, la zone où se trouvent réunies les conditions optima.

Dès le premier jaugeage et sur le vu des anomalies constatées, on pourra se corriger, modifier la nature de l'injection (ponctuelle ou répartie), agir sur la longueur du secteur de mesure et sur la durée d’injection, etc... Enfin, la connaissance approfondie du secteur d'opération au point de vue de l'aspect de l'écoulement et des variations diurnes du débit aux diverses époques de l'année permettra d'ajuster au mieux les techniques opératoires aux conditions locales.

Nous ne pouvons terminer cette étude d'ensemble sur la méthode chimique sans rappeler ici la technique opératoire de $M$. le Professeur Barbagelata (*) qui consiste, à partir de procédés d'injection de même nature que ceux que nous avons décrits, à provoquer une variation de conductibilité des eaux. Dans la section où normalement seraient faites les prises d'échantillons, l'eau est prélevée à l'aide d'une pompe et envoyée dans une cellule de mesure intercalée dans un pont à haute fréquence. On peut ainsi relever la courbe de passage du nuage, reconnaître sur celle-ci le palier de régime permanent et au moyen d'un étalonnage préalable, calculer le rapport des dilutions réalisées. Cette intéressante méthode susceptible de fournir des résultats immédiats, nécessite le transport à pied d'œuvre du matériel de dosage proprement dit. Nous avons voulu ici insister surtout sur le caractère de rusticité, la grande souplesse et la rapidité de la mise en œuvre de la méthode sur le terrain, réservant au laboratoire la technique plus délicate des dosages.

$\left(^{*}\right)$ Elettrotechnica, Fascicolo $n^{\circ} 5,15-2-1926$. - Magistrato Alle acque. Ufficio idrografico, Publicazione $\mathrm{n}^{\circ} 139,1935$. 


\section{CONCLUSION}

La méthode dite "chimique», de jaugeage des cours d'eau, d'origine ancienne, a fait l'objet de nouvelles études au cours des cinq dernières années et a repris une des premières places dans ce domaine.

Une connaissance encore imparfaite, mais moins confuse qu'autrefois, des conditions de propagation d'un nuage salin et de l'établissement d'un régime permanent, a permis de déterminer les longueurs de mélange, les limites du secteur de mesure, la durée du régime permanent à diverses abscisses et, dans l'ensemble, d'apporter les précisions nécessaires à une réalisation plus méthodique des jaugeages.

Parmi les sels solubles dont l'emploi est à préconiser, le bichromate de soude possède de précieux avantages, dont celui de relever de la méthode colorimétrique de dosage qui, tout en réclamant des soins, peut cependant être confiée à un personnel non spécialisé en analyse chimique.

Enfin, le gain très important réalisé sur lc poids et l'encombrement du matériel autorisc une rapide exécution des jaugeages dans les conditions d'accès les plus difficiles.

Dans l'ensemble, l'incertitude sur les débits mesurés par cette méthode est toujours inférieure à celle que l'on peut attendre du moulinet dans des conditions d'écoulement identiques el se range aux environs de $\pm 2 \%$ lorsque toutes les précautions ont été prises pour éviter les anomalies et les causes d'erreurs indiquées.

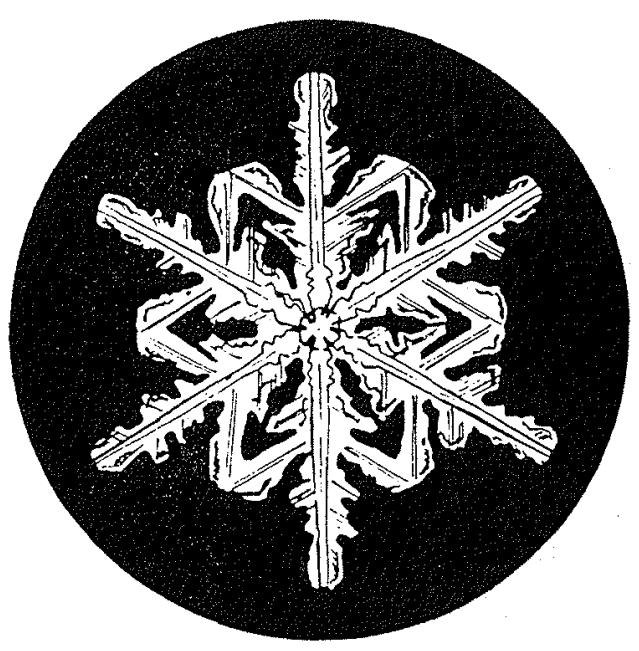

